\title{
Rendimiento de la evaluación clínica en el diagnóstico de fractura de huesos propios nasales*
}

\author{
Drs. PATRICIO ANDRADES C. ${ }^{1}$, CLAUDIO BOREL B. ${ }^{1}$, RICARDO BARTEL A. ${ }^{1}$, \\ RODRIGO HERNÁNDEZ N. ${ }^{1}$, RODRIGO VILLALOBOS A. ${ }^{1}$ \\ 1 Servicio de Cirugía Maxilofacial, Hospital del Trabajador de Santiago. \\ Santiago, Chile.
}

\begin{abstract}
Clinical assessment in the diagnosis of bone fractures in patients with nasal trauma

Introduction: The aim of this study was to evaluate the performance of clinical assessment in the diagnosis of bone fractures in patients with nasal trauma. Methods: Retrospective review of medical records of 220 patients with nasal trauma treated at the Maxillofacial Surgery Department of Hospital Workers in Santiago was performed. Eight clinical criteria were evaluated: epistaxis, ecchymosis, swelling, nasal injury, airway obstruction, nasal deviation, irregular nasal dorsum, and acute septal injury. The gold standard for diagnosis of nasal fracture was the radiological evaluation. The sensitivity, specificity, and positive/negative predictive value (PPV/NPV) were calculated for each separate clinical criteria (8), for all possible combinations of 2 clinical criteria (28) and 3 clinical criteria (56). Results: For any of the 8 criteria, the average sensitivity and negative predictive values for nasal fracture was very low $(<35 \%)$. However, the specificity and positive predictive values were relatively high (> 90\%) and increased, respectively, when at least one criterion was present (92\% and 94\% respectively), when 2 clinical criteria were present (98\% and 96\%, respectively), and when at least 3 clinical criteria were present (100\% for both). Conclusion: The presentation of the clinical criteria can be a valuable method for diagnosis of nasal fracture, however, when these clinical criteria are absent, the possibility of nasal fracture cannot be excluded, although the possibility is remote.
\end{abstract}

Key words: Nasal trauma, bone, fracture, clinical assessment, diagnostic.

\section{Resumen}

Introducción: El objetivo del presente trabajo fue conocer el rendimiento de la evaluación clínica en el diagnóstico de fractura de huesos propios en pacientes con trauma nasal. Material y Método: Revisión retrospectiva de fichas clínicas de 220 pacientes con trauma nasal atendidos en el Servicio de Cirugía Maxilofacial del Hospital del Trabajador de Santiago. Ocho criterios clínicos fueron evaluados: epistaxis, equimosis, inflamación, herida nasal, obstrucción de vía aérea, laterorrinia, dorso nasal irregular, y lesión aguda del tabique nasal. El estándar de oro para el diagnóstico de fractura nasal fue el estudio radiológico. La sensibilidad, especificidad, y positivo/valor predictivo negativo (VPP/VPN) se calcularon para cada criterio clínico por separado (8), para todas las combinaciones posibles de 2 criterios clínicos (28) y 3 criterios clínicos (56). Resultados: Para cualquiera de los 8 criterios, el promedio de sensibilidad y valor predictivo negativo

*Recibido el 12 de abril de 2012 y aceptado para publicación el 26 de mayo de 2012.

Los autores no declaran conflictos de interés.

Correspondencia: Dr. Patricio Andrades C.

Av. Vicuña Mackena 200, Santiago, Chile.

pandrades@u.uchile.cl 
para la fractura nasal fue muy baja $(<35 \%)$. Sin embargo, la especificidad y valores predictivos positivos fueron relativamente altas (> 90\%) y aumentó, respectivamente, cuando al menos un criterio estuvo presente (92\% y 94\%, respectivamente), cuando 2 criterios clínicos estaban presentes (98\% y 96\%, respectivamente), y cuando al menos 3 criterios clínicos estaban presentes (100\% para ambos). Conclusiones: La presentación de los criterios clínicos puede ser un método valioso para el diagnóstico de fractura nasal, sin embargo, cuando estos criterios clínicos están ausentes, la posibilidad de la fractura nasal no se puede descartar, aunque la posibilidad es remota.

Palabras clave: Trauma nasal, huesos propios, fractura, criterios clínicos, diagnóstico.

\section{Introducción}

La fractura de los huesos nasales es la lesión más común de los huesos de la cara y la tercera más frecuente de todas las fracturas del cuerpo. Se estima que el $40 \%$ de los casos de traumatismos faciales se asocian a fracturas de los huesos propios nasales ${ }^{1}$. De hecho, cada año en los Estados Unidos, aproximadamente 50.000 personas sufren una fractura de los huesos propios nasales ${ }^{2}$. Aunque la fractura nasal es considerada a menudo una lesión de menor importancia, un diagnóstico inadecuado y manejo tardío pueden producir importantes defectos funcionales y estéticos ${ }^{3,4}$.

El diagnóstico preciso de las fracturas nasales depende de una historia clínica y examen físico oportunos y completos ${ }^{5,6}$. Los pacientes suelen presentar una combinación de epistaxis, equimosis, deformidad, dolor, edema, la inestabilidad, y crepitación, sin embargo, estas características no siempre pueden estar presentes ${ }^{7}$. El estudio radiológico es el procedimiento estándar para confirmar el diagnóstico de fractura de huesos propios en pacientes con trauma nasal, a pesar de que su utilidad para la toma de decisiones clínicas es aún motivo de discusión ${ }^{8}$.

Para los médicos y especialistas con un alto grado de experiencia en el tratamiento de las fracturas nasales, un diagnóstico preciso a menudo se obtiene con sólo una historia clínica y examen físico minuciosos ${ }^{9}$. Por lo tanto, existe la posibilidad de identificar algunos elementos clínicos que efectivamente puedan ayudar a médicos generales y de emergencia en el diagnóstico correcto de fractura nasal basada principalmente en la evaluación clínica. El valor de los criterios clínicos para el diagnóstico de las fracturas nasales no ha sido formalmente evaluado. El propósito de esta investigación fue apreciar el rendimiento de la evaluación clínica en el diagnóstico de fractura de huesos propios en pacientes con trauma nasal.

\section{Material y Método}

\section{Diseño del estudio}

Se diseñó un estudio de cohorte retrospectivo con el fin de lograr los objetivos anteriormente mencionados. La información se recopiló a partir de expedientes clínicos obtenidos de la base de datos del
Servicio de Cirugía Maxilofacial en el Hospital del Trabajador de Santiago. El Hospital del Trabajador es un hospital terciario de referencia dedicado a la prevención, tratamiento y rehabilitación de accidentes de trabajo y sus secuelas.

\section{Selección de los participantes}

Todos los pacientes con traumatismo nasal vistos en el Departamento de Cirugía Maxilofacial del Hospital del Trabajador entre enero y diciembre del año 2004 se incluyeron en el estudio. En nuestra institución, todos los pacientes con traumatismo nasal se envían al Departamento de Cirugía Maxilofacial para la evaluación y tratamiento. Ninguna otra especialidad médica está involucrada en el manejo de estos pacientes. En nuestro departamento, el paciente con un traumatismo nasal se somete a un examen clínico minucioso con un protocolo especialmente diseñado para ello. También se someten a un completo estudio radiológico, que incluye radiografías de huesos propios en proyecciones laterales y anteroposteriores (radiografía de Waters). Dependiendo de la gravedad y el alcance de los hallazgos clínicos y radiológicos, también puede requerir una tomografía computarizada para completar el estudio diagnóstico. Toda la información es registrada en el sistema computacional del hospital.

\section{Métodos de medición}

La revisión se llevó a cabo en busca de la presencia o ausencia de los siguientes criterios clínicos: epistaxis (EPI, sangrado postraumático inmediato a través de una o ambas fosas nasales), equimosis (EQU, hematoma perinasal o periorbitario desarrollado poco después de un traumatismo nasal), herida nasal (HN, laceración post-traumático en la nariz producto del trauma), obstrucción de vía aérea (OVA, sensación subjetiva del paciente de obstrucción nasal que no estaba presente antes del trauma), inflamación nasal (INF, edema nasal desarrollado poco después de un trauma), laterorrinia ( $\mathrm{L}$, eje nasal desviado de línea media y la deformidad no estaba presente antes del trauma), irregularidad del dorso nasal (ID, palpación de espículas o depresiones en las paredes anteriores y laterales de la nariz) y lesión aguda del tabique nasal (LSA, observación de un hematoma o una laceración del tabique nasal 
durante la nasoscopia). La revisión de las historias clínicas fue realizada por dos investigadores independientes. Los resultados se consideraron positivos o negativos para el análisis de los datos. Los datos obtenidos en relación a los 8 criterios clínicos estudiados fueron puestos en un archivo de Excel para su análisis posterior. El estándar de oro para el diagnóstico definitivo de fractura de hueso nasal, fue el informe de las radiografías o resultados de la tomografía dadas por el radiólogo, que era ciego a la evaluación clínica del paciente. Un investigador independiente realizó el análisis estadístico.

\section{Análisis de datos}

Las frecuencias se utilizaron para resumir las variables categóricas. Un total de 92 comparaciones entre los datos clínicos y el patrón de oro se realizaron con un criterio clínico (8), con todas las combinaciones posibles de dos criterios clínicos (28 combinaciones sin repetición), y con 3 criterios clínicos (56 combinaciones sin repetición). La sensibilidad, especificidad, valor predictivo positivo (VPP) y negativo (VPN) se calcularon para cada comparación. Además, un análisis de regresión logística múltiple se utilizó para evaluar la capacidad combinada de los criterios clínicos para diagnosticar una fractura del hueso nasal, y estimar el Odds Ratio (OR) con un intervalo de confianza del 95\% (IC). Los datos fueron analizados mediante el paquete estadístico SPSS versión 17.0 (Chicago, IL).

\section{Resultados}

Un total de 220 pacientes con traumatismo nasal fueron evaluados en el Departamento de Cirugía Oral y Maxilofacial durante el período de estudio. Hubo 157 hombres (71,4\%) y 63 mujeres (28,6\%), con una edad media de $36 \pm 10,7$ años. La fractura del hueso nasal se diagnosticó en 167 pacientes (76\%), y en 53 pacientes (24\%) no había más que una contusión nasal sin lesión ósea. Las principales causas de traumatismo nasal fueron accidente automovilístico (35\%), traumatismo por un objeto contundente (20\%) y una caída accidental (17\%). Sólo 29 pacientes (13\%) tenían comorbilidades y 20 pacientes (9\%) presentaron un trauma nasal previo. Veintidós pacientes $(10 \%)$ tenían una fractura de otro hueso facial (41\% dentoalveolar, 18\% frontoorbitaria y fractura extendida de tercio medio facial $14 \%)$ y 29 (13\%) tuvieron un trauma asociado en otras partes del cuerpo (cerebro 48\%, la extremidad superior 35\%, y cuello 14\%).

La prevalencia, sensibilidad, especificidad, VPN y VPP de los criterios clínicos se muestran en la Tabla 1. La sensibilidad y VPN, cuando sólo un criterio clínico estuvo presente, fue de 34\% y 32\% respectivamente. El criterio clínico con la mayor sensibilidad y VPN fue la epistaxis (69\% y 50\% respectivamente). La especificidad y VPP, cuando sólo un criterio clínico estuvo presente, fue del 92\% y $94 \%$ respectivamente. El criterio clínico de mayor especificidad y VPP fue la lesión aguda del tabique nasal (100\% y 100\%), seguida por equimosis (98\% y 98\%), obstrucción de vía aérea (96\% y 96\%), y laterorrinia (96\% y 96\%).

La prevalencia, sensibilidad, especificidad, VPN y VPP para todas las combinaciones de dos criterios clínicos y la combinación general de los 3 criterios clínicos se muestran en la Tabla 2. La tasa de sensibilidad global para la detección de fractura nasal cuando dos criterios clínicos estaban presentes al mismo tiempo, fue del $11 \%$ y el VPN del 26\%. Sin embargo, la especificidad y VPP fueron $98 \%$ y $96 \%$ respectivamente. Cuando 3 criterios clínicos estaban presentes al mismo tiempo, la sensibilidad y VPN se mantuvieron muy bajos (6\% y $25 \%$ ) pero la especificidad y VPP alcanzaron el $100 \%$.

La estimación del riesgo de fractura de hueso nasal de acuerdo a criterios clínicos se muestra en la Tabla 3. En el análisis de regresión logística, los

Tabla 1. Distribución de frecuencias y rendimiento de cada uno de los criterios clínicos estudiados

\begin{tabular}{|lrccccc|}
\hline Criterio clínico & Porcentaje (n) & Sensibilidad & Especificidad & VPP & VPN \\
\hline Epistaxis (EPI) & $54 \%$ & $(119)$ & $69 \%$ & $94 \%$ & $97 \%$ & $50 \%$ \\
\hline Equimosis (EQU) & $20 \%$ & $(44)$ & $26 \%$ & $98 \%$ & $98 \%$ & $30 \%$ \\
\hline Herida nasal (HN) & $42 \%$ & $(92)$ & $46 \%$ & $70 \%$ & $83 \%$ & $29 \%$ \\
Obstrucción vía aérea (OVA) & $15 \%$ & $(33)$ & $19 \%$ & $96 \%$ & $94 \%$ & $27 \%$ \\
\hline Inflamación (INF) & $45 \%$ & $(98)$ & $56 \%$ & $92 \%$ & $96 \%$ & $40 \%$ \\
Laterorrina (L) & $15 \%$ & $(32)$ & $18 \%$ & $96 \%$ & $94 \%$ & $27 \%$ \\
\hline Irregularidad dorso nasal (ID) & $24 \%$ & $(52)$ & $29 \%$ & $92 \%$ & $92 \%$ & $29 \%$ \\
Lesión septal aguda (LSA) & $8 \%$ & $(17)$ & $10 \%$ & $100 \%$ & $100 \%$ & $26 \%$ \\
Promedio & $28 \%$ & & $34 \%$ & $92 \%$ & $94 \%$ & $32 \%$ \\
\hline
\end{tabular}


RENDIMIENTO DE LA EVALUACIÓN CLÍNICA EN EL DIAGNÓSTICO DE FRACTURA DE HUESOS PROPIOS NASALES

Tabla 2. Distribución de frecuencias y rendimiento de los criterios clínicos agrupados

\begin{tabular}{|c|c|c|c|c|c|c|}
\hline \multirow{2}{*}{$\begin{array}{l}\text { Criterios clínicos } \\
\text { EPI+EQU }\end{array}$} & \multicolumn{2}{|c|}{ Prevalencia (Nn) } & \multirow{2}{*}{$\begin{array}{c}\text { Sensibilidad } \\
19 \%\end{array}$} & \multirow{2}{*}{$\begin{array}{c}\text { Especificidad } \\
100 \%\end{array}$} & \multirow{2}{*}{$\begin{array}{c}\mathbf{V P P} \\
100 \%\end{array}$} & \multirow{2}{*}{$\begin{array}{l}\text { VPN } \\
28 \%\end{array}$} \\
\hline & $14 \%$ & (31) & & & & \\
\hline $\mathrm{EPI}+\mathrm{HN}$ & $23 \%$ & (50) & $30 \%$ & $100 \%$ & $100 \%$ & $31 \%$ \\
\hline $\mathrm{EPI}+\mathrm{OVA}$ & $12 \%$ & (26) & $10 \%$ & $100 \%$ & $100 \%$ & $27 \%$ \\
\hline EPI+INF & $16 \%$ & (35) & $21 \%$ & $100 \%$ & $100 \%$ & $29 \%$ \\
\hline EPI+L & $11 \%$ & (24) & $14 \%$ & $98 \%$ & $96 \%$ & $27 \%$ \\
\hline EPI+ID & $17 \%$ & (37) & $22 \%$ & $98 \%$ & $97 \%$ & $28 \%$ \\
\hline EPI+LSA & $7 \%$ & (15) & $9 \%$ & $100 \%$ & $100 \%$ & $26 \%$ \\
\hline INF+EQU & $14 \%$ & (31) & $18 \%$ & $98 \%$ & $97 \%$ & $28 \%$ \\
\hline $\mathrm{INF}+\mathrm{HN}$ & $22 \%$ & (48) & $26 \%$ & $92 \%$ & $92 \%$ & $28 \%$ \\
\hline INF+OVA & $10 \%$ & (22) & $12 \%$ & $96 \%$ & $91 \%$ & $26 \%$ \\
\hline $\mathrm{INF}+\mathrm{L}$ & $7 \%$ & (16) & $10 \%$ & $100 \%$ & $100 \%$ & $26 \%$ \\
\hline INF+ID & $14 \%$ & (31) & $17 \%$ & $96 \%$ & $94 \%$ & $27 \%$ \\
\hline INF+LSA & $5 \%$ & (6) & $6 \%$ & $100 \%$ & $100 \%$ & $25 \%$ \\
\hline HN+EQU & $10 \%$ & (21) & $12 \%$ & $98 \%$ & $95 \%$ & $26 \%$ \\
\hline $\mathrm{HN}+\mathrm{OVA}$ & $7 \%$ & (15) & $8 \%$ & $96 \%$ & $87 \%$ & $25 \%$ \\
\hline $\mathrm{HN}+\mathrm{L}$ & $5 \%$ & (11) & $7 \%$ & $100 \%$ & $100 \%$ & $25 \%$ \\
\hline $\mathrm{HN}+\mathrm{ID}$ & $13 \%$ & (29) & $16 \%$ & $96 \%$ & $93 \%$ & $27 \%$ \\
\hline $\mathrm{HN}+\mathrm{LSA}$ & $4 \%$ & (8) & $5 \%$ & $100 \%$ & $100 \%$ & $25 \%$ \\
\hline ID+EQU & $8 \%$ & (17) & $10 \%$ & $98 \%$ & $94 \%$ & $26 \%$ \\
\hline ID+OVA & $8 \%$ & (18) & $10 \%$ & $96 \%$ & $89 \%$ & $25 \%$ \\
\hline ID+L & $6 \%$ & (14) & $7 \%$ & $96 \%$ & $86 \%$ & $25 \%$ \\
\hline ID+LSA & $3 \%$ & (6) & $4 \%$ & $100 \%$ & $100 \%$ & $25 \%$ \\
\hline EQU+OVA & $5 \%$ & (10) & $5 \%$ & $98 \%$ & $90 \%$ & $25 \%$ \\
\hline $\mathrm{EQU}+\mathrm{L}$ & $3 \%$ & (6) & $4 \%$ & $100 \%$ & $100 \%$ & $25 \%$ \\
\hline EQU+LSA & $2 \%$ & (4) & $2 \%$ & $100 \%$ & $100 \%$ & $25 \%$ \\
\hline $\mathrm{OVA}+\mathrm{L}$ & $5 \%$ & (11) & $7 \%$ & $100 \%$ & $100 \%$ & $25 \%$ \\
\hline OVA+LSA & $2 \%$ & (5) & $3 \%$ & $100 \%$ & $100 \%$ & $25 \%$ \\
\hline ND+LSA & $2 \%$ & (5) & $3 \%$ & $100 \%$ & $100 \%$ & $25 \%$ \\
\hline Promedio 2 criterios & $9 \%$ & & $11 \%$ & $98 \%$ & $96 \%$ & $26 \%$ \\
\hline Promedio 3 criterios & $4 \%$ & & $6 \%$ & $100 \%$ & $100 \%$ & $25 \%$ \\
\hline
\end{tabular}

Tabla 3. Estimación del riesgo de fractura nasal según los criterios clínicos

\begin{tabular}{|lccccc|}
\hline & $\begin{array}{c}\text { Coeficiente de } \\
\text { regresión }\end{array}$ & $\begin{array}{c}\text { Chi cuadrado } \\
\left(\boldsymbol{\chi}^{2}\right)\end{array}$ & $\begin{array}{c}\text { Odds Ratio } \\
(\mathbf{O R})\end{array}$ & $\begin{array}{c}\text { Intervalo de confianza } \\
\mathbf{9 5 \%}\end{array}$ \\
\hline Intersección & $-23,855$ & 0,000 & & & 7,090 \\
Irregularidad dorso nasal & 0,459 &, 548 & 1.583 & 0,354 & 91,180 \\
Epistaxis & 3,211 & 0,000 & 24.809 & 6,751 & 79,134 \\
\hline Equimosis & 2,192 & 0,049 & 8.953 & 1,013 & 5,773 \\
Herida nasal & 0,847 & 0,067 & 2.333 & 0,943 & 19,898 \\
Inflamación & 1,790 & 0,003 & 5.987 & 1,801 & 14,046 \\
\hline Laterorrinia & 0,747 & 0,440 & 2.110 & 0,317 & 8,627 \\
\hline Obstrucción vía aérea & 0,007 & 0,995 & 1.007 & 0,118 & 1,461 \\
\hline Lesión septal aguda & 15,654 & 0,017 & 1.272 & 1,107 & \\
\hline
\end{tabular}


únicos factores clínicos que predicen significativamente la aparición de una fractura de hueso nasal fueron epistaxis (OR: 24,8, IC 95\%: 6,8-91,2), equimosis (OR: 9.0, IC 95\%: 1,0-79,1), la inflamación (OR: 6.0, IC 95\%: 1,8 a 19,9) y la lesión septal aguda (OR: 1.3, IC 95\%: 1,1-1,5).

\section{Discusión}

El rendimiento de los criterios clínicos para el diagnóstico de fractura nasal se midió utilizando la sensibilidad, especificidad, VPP y VPN. La sensibilidad de una prueba se define como la proporción de personas con la enfermedad que tienen un resultado positivo; la especificidad es la proporción de personas sin la enfermedad que tienen un resultado negativo; el VPP es la proporción de aquellos con una prueba positiva que realmente tienen la enfermedad; y el VPN es la proporción de aquellos con una prueba negativa que no tienen la enfermedad. La sensibilidad y especificidad son medidas importantes de la precisión diagnóstica de una prueba, pero no pueden ser utilizadas para estimar la probabilidad de la enfermedad en cada paciente individualmente. Por el contrario, el VPP y VPN proporcionan estimaciones de la probabilidad de la enfermedad, pero ambos parámetros dependen de la prevalencia de la enfermedad y pueden variar en consecuencia. Mientras que la sensibilidad y la especificidad son medidas importantes de la precisión diagnóstica de una prueba, no son de uso práctico en el ámbito clínico, es decir, no ayuda a los médicos en la estimación de la probabilidad de la enfermedad. Para este propósito, el VPP y el VPN tienen una mayor utilidad y son más apropiados ${ }^{10}$.

Los resultados de nuestro estudio demostraron que la sensibilidad promedio para cualquier criterio clínico usado en forma aislada fue baja (34\%) y disminuyó aún más cuando 2 ó 3 criterios estaban presentes (11 y 6\% respectivamente). Esto quiere decir que de todos los pacientes con fractura nasal, menos del 34\% presenta 1 o más de los síntomas y signos estudiados. Como la sensibilidad hace referencia a cuan bueno es un examen en detectar los pacientes que están realmente enfermos, se podría decir que la evaluación clínica en pacientes con trauma nasal, no es un test adecuado para detectar los pacientes que tienen realmente una fractura de huesos propios nasales. Por otro lado, la especificidad promedio para cualquier criterio clínico aislado fue muy alta (92\%), y aumentó aún más cuando 2 ó 3 criterios se usaron en forma combinada (98 y100\% respectivamente). Esto quiere decir que de los pacientes sin fractura nasal, más del $92 \%$ no presenta los síntomas y signos estudiados. Como la especificidad hace referencia a la capacidad de un examen de detectar los pacientes que están verdaderamente sanos, se podría afirmar que la evaluación clínica en pacientes con trauma nasal es un excelente test para detectar los pacientes que realmente no tienen una fractura de huesos propios nasales (examen de descarte).

Lamentablemente desde el punto de vista clínico esto no es de gran utilidad debido a que cuando el paciente llega a la consulta lo hace con el examen positivo o negativo, y nosotros desconocemos si el paciente está enfermo o no. Lo que le interesa al clínico es intentar predecir la presencia o ausencia de enfermedad utilizando los elementos clínicos y para ello el VPP y VPN son de mayor utilidad. En nuestro estudio el VPN presentó valores bajos cuando 1 o más criterios fueron utilizados. Esto quiere decir que de todos los pacientes sin signos clínicos, menos del 32\% presentó ausencia de una fractura nasal. Por este motivo, la ausencia de algún elemento clínico en un paciente con trauma nasal no asegura la ausencia de una fractura de huesos propios. Por el contrario, el VPP presentó valores elevados en todas las comparaciones realizadas. Es decir, que de todos los pacientes con una evaluación clínica positiva, más del 92\% presentó una fractura nasal. Por lo tanto, la presencia de 1 o más de los elementos clínicos estudiados asegura, en un alto porcentaje, la presencia de una fractura de huesos propios.

Hay consenso en que el VPP tiene la mayor utilidad para los clínicos en la estimación de la probabilidad de enfermedad ${ }^{10}$, por lo que esta medida debe tener la mayor importancia clínica. Nosotros pudimos observar que en promedio, cuando al menos sólo 1 criterio clínico está presente, la probabilidad de fractura nasal real es de $94 \%$, cuando al menos 2 criterios clínicos están presentes al mismo tiempo, esta probabilidad es de $96 \%$, y finalmente, cuando por lo menos 3 criterios clínicos están presentes al mismo tiempo, esta probabilidad es del $100 \%$. Sin embargo, hay pacientes sin los criterios clínicos estudiados presentes después de un trauma nasal que igual podrían tener una fractura de los huesos propios. Como lo mencionamos anteriormente, el bajo VPN de estos resultados indica que cuando los criterios clínicos están ausentes, los médicos no pueden descartar la presencia de una fractura nasal. Sin embargo, sobre la base de la fórmula de regresión logística calculado en el presente estudio, donde " $\mathrm{y}=0,03+0,47 \mathrm{EPI}+0,27 \mathrm{EQU}+0,36 \mathrm{INF}$ $+0,26$ LSA", podemos ver que aunque es posible, es muy difícil tener una fractura nasal (y) sin criterios clínicos asociados. Esta probabilidad está dada por la constante igual a 0,03 en la fórmula. Además, 
de los 220 pacientes evaluados, sólo 6 (2,73\%) tuvieron una fractura nasal sin criterios clínicos asociados.

Varios estudios han demostrado que el diagnóstico de fractura de huesos propios nasales puede ser realizado correctamente sólo con la evaluación clínica ${ }^{5,6}$. Por ejemplo, la presencia de epistaxis después de un trauma nasal ha demostrado estar asociada con un riesgo significativamente mayor de deformidad nasal externa ${ }^{11}$. Dentro de los elementos clínicos estudiados, los únicos síntomas y signos que aumentan en forma estadísticamente significativa el riesgo de fractura nasal fueron la epistaxis, inflamación, equimosis, y lesión septal aguda. La lesión septal aguda fue el único criterio clínico con los valores máximos para la especificidad (100\%) y VPP (100\%). Por lo tanto, si hay una LSA, siempre habrá una fractura nasal asociada. Sin embargo, de todos los criterios clínicos considerados en el análisis, la LSA requiere de cierta experiencia por parte del médico en el examen interno de la nariz a través de una especuloscopia. Por el contrario, la epistaxis presentó el OR más alto y excelentes índices de rendimiento para el diagnóstico de fractura nasal (Sensibilidad 69\%, Especificidad 94\%, VPP $97 \%$ y VPN 50\%). Es un elemento clínico muy fácil de objetivar y el alto VPP para este criterio clínico indica una alta probabilidad de fractura nasal real. El primer síntoma que se debiera preguntar a todo paciente con trauma nasal debiera ser la presencia de sangramiento nasal. Esta simple pregunta podría reducir dramáticamente los costos y tiempos de espera en estos pacientes.

\section{Referencias}

1. Reilly M, Davidson S. Open vs closed approach to the nasal pyramid for fracture reduction. Arch Fac Plast Surg. 2007;9:82-6.

2. Guyuron B, Zarandy S. Does rhinoplasty make the nose more susceptible to fracture? Plast Reconstr Surg. 1994;93:313-7.

3. Carvalho TB, Cancian LR, Marques CG, Piatto VB, Maniglia JV, Molina FD. Six years of facial trauma care: an epidemiological analysis of 355 cases. Braz J Otorhinolaryngol. 2010;76:565-74.

4. Verwoerd CDA. Present day treatment of nasal fractures: Closed versus open reduction. Facial Plast Surg. 1992;8:220-3.

5. Rohrich R, Adams W. Nasal Fracture Management: Minimizing secondary nasal deformity. Plast Reconstr Surg. 2000;106:266-73.

6. Rubinstein B, Bradley ES. Management of Nasal Fractures. Arch Fam Med. 2000;9:738-42.

7. Tremolet de Villers Y, Schultz RC. Nasal fractures. J Trauma 1975;15:319-27.

8. Gürkov R, Clevert D, Krause E. Sonography versus plain $\mathrm{x}$ rays in diagnosis of nasal fractures. Am J Rhinol. 2008;22:613-6.

9. Perkins SW, Dayan SH. Management of Nasal Trauma. Aesth Plast Surg. 2002;26:S3.

10. Akobeng AK. Understanding diagnostic tests 1: sensitivity, specificity and predictive values. Acta Paediatr. 2007;96:338-41.

11. Daniel M, Raghavan U. Relation between epistaxis, external nasal deformity, and septal deviation following nasal trauma. Emerg Med J. 2005;22:778-9. 